\title{
Solenoid actuated five-fingered robotic hand design: evaluating the capability of solenoid actuators in generating basic finger motions
}

\author{
Syed Zainal Abidin Syed Kamarul Bahrin ${ }^{1}$, Khairul Salleh Mohamed Sahari ${ }^{2}$ \\ ${ }^{1}$ Department of Electrical Power Engineering, Universiti Tenaga Nasional, Malaysia \\ ${ }^{2}$ Department of Mechanical Engineering, Universiti Tenaga Nasional, Malaysia
}

\begin{tabular}{l} 
Article Info \\
\hline Article history: \\
Received Jan 3, 2019 \\
Revised Apr 6, 2019 \\
Accepted Apr 28, 2019 \\
\hline
\end{tabular}

Keywords:

Five-fingered robotic hand

Linear actuator

Robotic hand

Robotics

Solenoid

\begin{abstract}
There are numerous robotic hand designs but the five-fingered robotic hand design is the most dexterous robotic hand design due to its similar appearance and motions with the human hands. The fingers' motions are commonly driven by geared motors or other types of emerging technologies. However, the motions are yet to be driven directly by solenoid actuators due to its limited stroke length and also general perceptions of its applications as an actuator. Nevertheless, solenoid actuators are known for their fast reaction time and strong holding force which are useful to generate fast motions and strong grasping actions. To realise this concept, previous research and developments had been done by the authors but the outcome was not convincing enough due to the absence of a working prototype. Therefore, this paper introduced a new solenoid actuated robotic hand design and its prototype. The prototype was then tested and evaluated in accordance to the operational concept where the findings showed that the solenoid actuators were capable of generating basic finger motions.
\end{abstract}

Copyright $\odot 2019$ Institute of Advanced Engineering and Science. All rights reserved.

\section{Corresponding Author:}

Syed Zainal Abidin Syed Kamarul Bahrin,

Department of Electrical Power Engineering,

Universiti Tenaga Nasional,

Jalan Ikram-Uniten, 43000, Kajang, Selangor, Malaysia.

Email: SZainal@uniten.edu.my

\section{INTRODUCTION}

\subsection{Overview}

Robotics is a technology that consists of processes or actions that are involved in designing, constructing, operating and developing robots [1]. The technology also includes the computer systems for controlling, sensory feedback and information processing of robots. These technologies are very important in performing a wide range of tasks that are very difficult or impossible to be executed solely by humans due to various hazardous elements or safety issues [2-3] such as performing dangerous manufacturing processes, handling biohazard elements, disarming bombs, post-disaster search and rescue missions, etc. There are several robotic components involved to perform these tasks but the most utilised component is the robot's hand because it has more degrees of freedom (DOF) compared to the other components.

Robotic hand is available in a wide range of designs but the five-fingered robotic hand design has the highest number of DOF. This is because a five-fingered robotic hand is designed to be more dexterous compared to the other robotic hand designs as it imitates a human hand in term of appearances and motions. These motions are usually generated by electric motors [4-14], but recent advancement in actuator technologies such as shape-memory alloy [15-16], pneumatic actuators [14, 17-18] and hydraulic actuators [19-21] are becoming more common in generating motions for a five-fingered robotic hand design. Even with these technological advancements, modern technological developments of a five-fingered robotic 
hand have yet to explore the option of using solenoid actuator as the main driving force for the motions. This is due to the common perceptions or understandings of the solenoid applications as an actuator.

Solenoids are generally utilised as actuators for valve control [22-23] and switching applications [24-25] due to the large force that the solenoids can produce at short stroke length whilst having a small reaction time. This characteristic is very useful to provide strong grasping strength and fast motions for any five-fingered robotic hand design. However, the limited displacement due to the short stroke length will cause a narrow range of motions for the five-fingered robotic hand design. Nevertheless, the developments of solenoid designs have improved over the past few years [26] that allow the solenoid to have a longer displacement while maintaining a decent, same or higher amount of force albeit at a higher cost.

\subsection{Solenoid Applications in Robotics}

Solenoid applications are quite limited but the production of solenoids is abundant due to the high demand in controlling and switching applications for domestic and industrial purposes. Since robotics also involves in a lot of controlling and switching applications, solenoid technology is bound to appear in robotics developments and ultimately applied in the system. Based on the recent research and literature reviews that have been done, solenoids were never used to generate motions for the robotic fingers directly but are used for other robotic applications such as locking mechanism, pneumatic valve control and human arm motions.

\subsubsection{Locking Mechanism}

The locking mechanism is used to allow an under-actuated robotic hand to perform multiple grasps [27] and generate a lot of independent degrees of freedom from a single actuator [28]. The multiple grasps concept were realised by developing three special transmissions to allow the compact joint connected motion which connected motion among fingers and adduction/abduction. These specially designed transmissions reduced the volume and weight of the hand while at the same time maintain functions necessary for generating desired grasps. The locking mechanism can select three different opposed positions of the thumb to generate four different grasp actions.

Meanwhile, the later locking mechanism is applied for linear One-to-Many (OTM) system that allows a single actuator to store energy in springs once the solenoids are latched to the toothed pulleys. The energy stored in the springs are later used to drive multiple actuator units or independently controlled mechanical degrees of freedom once the solenoids are not latched to the toothed pulleys.

\subsubsection{Pneumatic Valve Control}

The pneumatic valve of a direct-injection liquid monopropellant powered actuation system is controlled by solenoid to provide mechanical power to self-powered human-scale robots [29]. The pneumatic-type actuators are powered by a hot gas generator when the actuation system triggers the catalytic decomposition of a monopropellant. To be precise, the solenoid injection valves control the flow of the monopropellant via a catalyst pack into the specified sides of the cylinder which subsequently generates pressure on the pneumatic actuator. In order to depressurise the pneumatic actuator, a three-way proportional spool valve will exhaust one of the two cylinder chambers. It can be seen that this system can generate more energetic figure as compared to a battery-powered servomotor.

\subsubsection{Human Arm Motions}

The arm motions are controlled by multi-stage solenoids to generate high amount of force at long stroke to imitate an arm muscle motions [30] and realising an exoskeleton concept for rehabilitation called Flexible Solenoid-Coil Artificial Muscle (FSAM) [31]. High amount of force and long stroke are necessary to generate proper arm functionality because moving an arm requires at least $10 \mathrm{kN}$ of force and $80 \mathrm{~mm}$ of displacement.

\subsection{Solenoid as Main Actuator for Robotic Hand Design}

As stated previously, solenoid actuators were never used directly or operate as prime movers to generate motions for a five-fingered robotic hand due to its short stroke length limitation. However, previous research and development that had been done by the authors in [32] showed that it was possible to generate motions for a five-fingered robotic hand by using multiple solenoid actuators and unique mechanical linkages design. The outcome of the research was a five-fingered robotic hand design that is powered by ten solenoid actuators that can work independently or in tandem to generate basic motions of a finger. However, the outcome was based solely on mechanical theories or motional concept rather than on a working prototype. Therefore, this paper will firstly introduce a new solenoid actuated five-fingered robotic hand design and its operational concept in generating basic finger motions. The prototype will then be introduced and tested in 
accordance to the operational concept so that the solenoid actuators' capability in generating basic finger motions can be evaluated.

\subsubsection{Overall Design}

A few design modifications on the previous design in [32] were performed to develop a new solenoid actuated five-fingered robotic hand design that is shown in Figure 1 with and without its TPU covers. The design modifications were specifically made to improve the joints' flexibility by using thermoplastic polyurethane (TPU) material for the hinge and to ease the wiring routing/connections by relocating the sensors to the top of the fingers. The modifications were also specifically made to protect the sensors and high tension strings by using TPU covers at the fingers, palm and backhand. Both designs are, in approximately, based on an average human (male) hand size (length $=189 \mathrm{~mm}$, width $=84 \mathrm{~mm}$ ) [33].

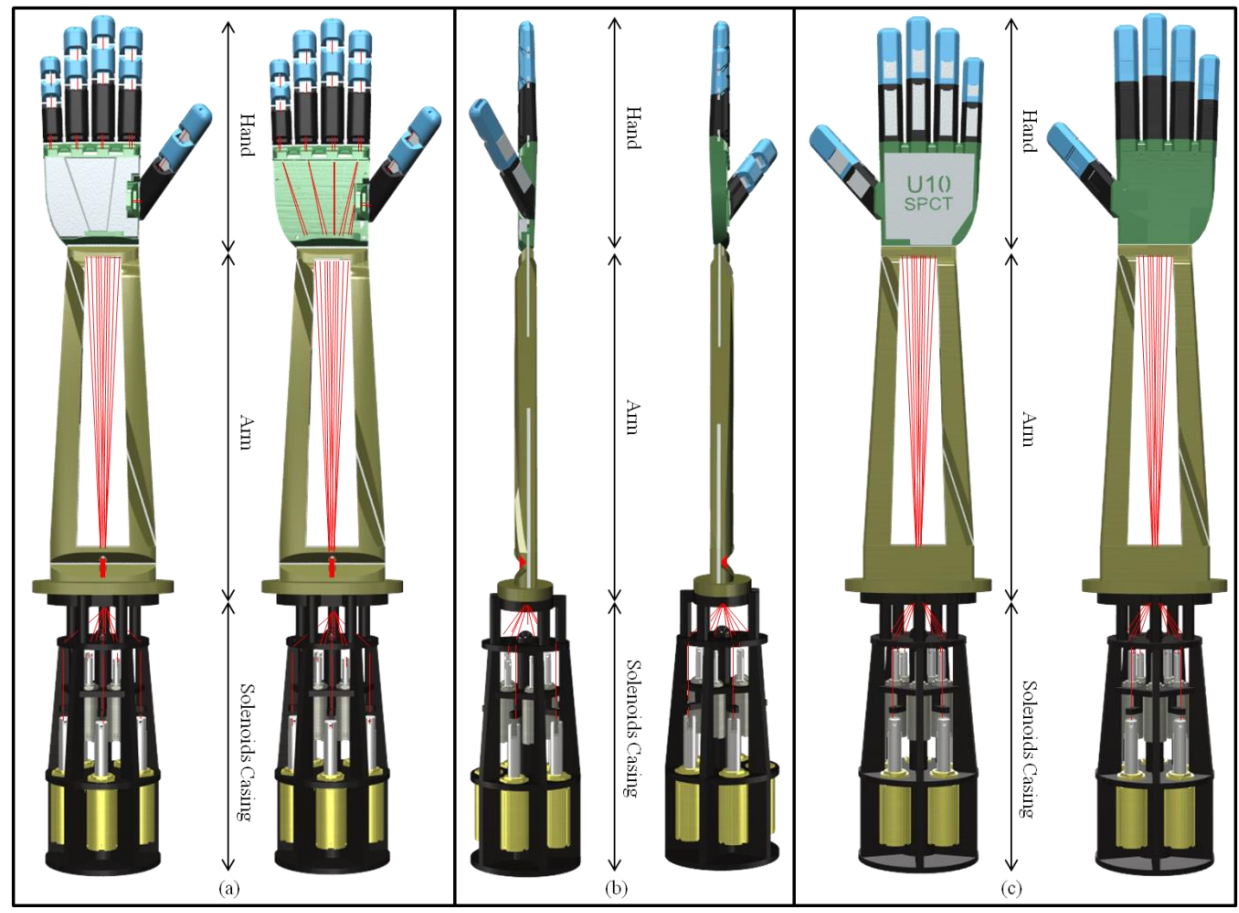

Figure 1. The solenoid actuated five-fingered robotic hand design (a) palm (b) sides and (c) backhand view.

The red lines represent the high tension string connections between the fingers and solenoid actuators

The overall design is divided into three main parts; hand, arm and solenoids casing. These parts are connected to one another via joints to improve flexibility and durability during experimentations. The arm also has internal joints in its design (the diagonal grooves) to improve flexibility and durability.

It should be noted that the motions are only generated by the hand and solenoids casing parts because the solenoid actuators will pull the high tension strings that are connected to the fingers. The arm will only provide passage for the strings. Thus, the following sections will provide brief descriptions on the hand and solenoids casing components.

\subsubsection{Hand Components}

The hand components are shown in Figure 2, with each component's function is described briefly in Table 1. It is important to note that the TPU covers are not shown and the index finger is made transparent to display the high tension strings connections.

The hand design generally consists of fingers that are divided into three phalanges, palm/backhand and also passages that guide the strings. Since the phalanges are connected to the palm/backhand and also to each another through hinges, the fingers can move in unidirectional motion during actuation (each phalange can move or rotate at a maximum of $90^{\circ}$ ). It is important to mention that the fundamental mechanism or operation for each finger is identical even though the thumb's bottom phalange design is slightly different. 


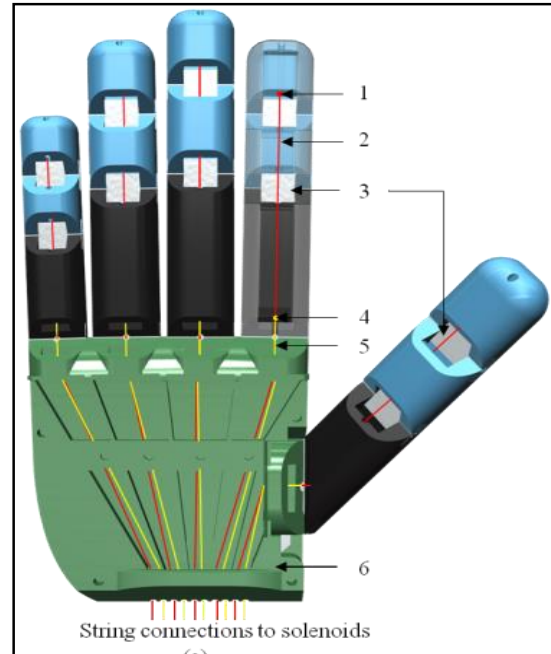

(a)
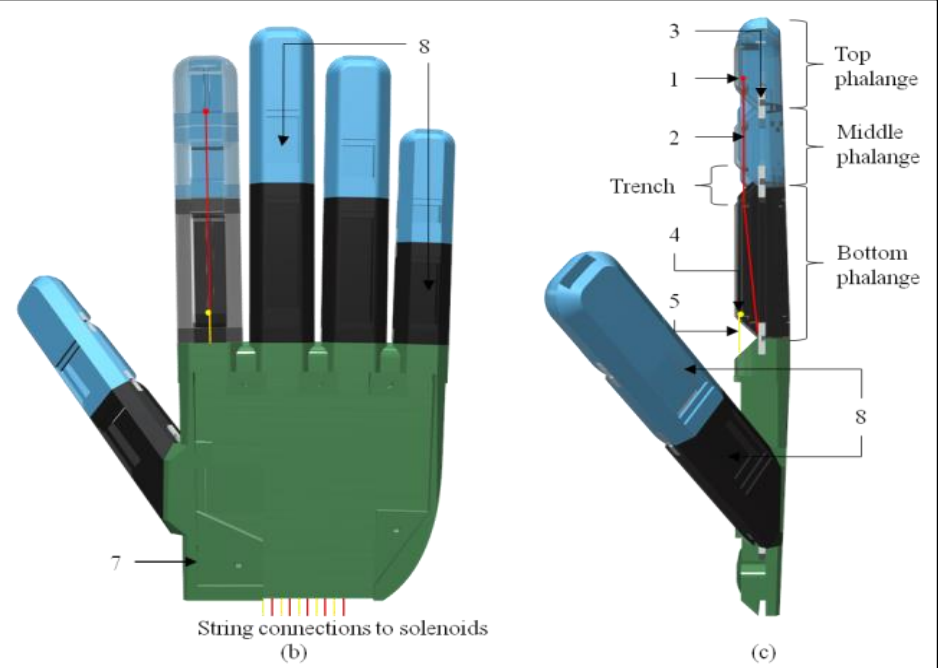

Figure 2. (a) Palm, (b) backhand and (c) side view of the hand components

Table 1. Labels for Figure 2

\begin{tabular}{|c|c|c|}
\hline No & Description & Function \\
\hline 1 & String stopper (double phalanges) & Locks the braided nylon to move the top and middle phalanges during actuation. \\
\hline 4 & String stopper (single phalange) & Locks the braided nylon to move the bottom phalange during actuation. \\
\hline 5 & High tension string (single phalange) & $\begin{array}{l}\text { Braided nylon that is connected to a solenoid actuator to move the bottom } \\
\text { phalange from a single actuation. }\end{array}$ \\
\hline 8 & Sensor housing & $\begin{array}{l}\text { Positions the feedback sensors and wiring connections appropriately when the } \\
\text { finger moves. }\end{array}$ \\
\hline
\end{tabular}

\subsubsection{Solenoids Casing Components}

The solenoids casing components are shown in Figure 3 and the function for each component is described in Table 2 .

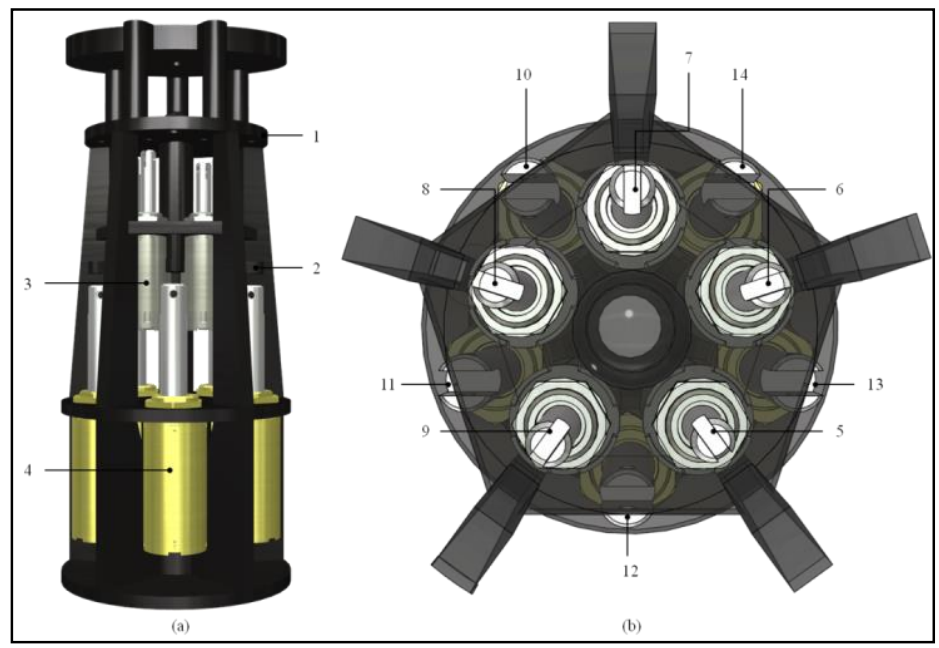

Figure 3. Solenoids casing (a) side and (b) top view 
Table 2. Labels for Figure 3.

\begin{tabular}{rll}
\hline No & Description & Function \\
\hline 1 & $\begin{array}{l}\text { Solenoid armature stopper. } \\
\text { (single phalange) }\end{array}$ & $\begin{array}{l}\text { The end stop for the single phalange solenoid actuator that ensures the armature does } \\
\text { not dislodged from the solenoid's casing. } \\
\text { The end stop for the double phalanges solenoid actuator that ensures the armature does } \\
\text { not dislodged from the solenoid's casing. }\end{array}$ \\
& $\begin{array}{l}\text { Solenoid armature stopper. } \\
\text { (double phalanges) }\end{array}$ & $\begin{array}{l}\text { Solenoid actuator (single phalange) } \\
\text { Actuator for the single phalange system. }\end{array}$ \\
4 & Solenoid actuator (double phalanges) & Actuator for the double phalanges system. \\
5 & Single phalange - thumb & Pulls the braided nylon to move the thumb's bottom phalange. \\
6 & Single phalange - index finger & Pulls the braided nylon to move the index finger's bottom phalange. \\
7 & Single phalange - middle finger & Pulls the braided nylon to move the middle finger's bottom phalange. \\
8 & Single phalange - ring finger & Pulls the braided nylon to move the ring finger's bottom phalange. \\
9 & Single phalange - small finger & Pulls the braided nylon to move the small finger's bottom phalange. \\
10 & Double phalanges - thumb & Pulls the braided nylon to move the thumb's middle and top phalanges. \\
11 & Double phalanges - index finger & Pulls the braided nylon to move the index finger's middle and top phalanges. \\
12 & Double phalanges - middle finger & Pulls the braided nylon to move the middle finger's middle and top phalanges. \\
13 & Double phalanges - ring finger & Pulls the braided nylon to move the ring finger's middle and top phalanges. \\
14 & Double phalanges - small finger & Pulls the braided nylon to move the small finger's middle and top phalanges.
\end{tabular}

A basic solenoid actuator, which is shown in Figure 4, is used to move the phalange(s) by pulling the high tension string through the plunger when current passes through the copper windings. For this particular robotic hand design, there are ten solenoids; five large and five small solenoid actuators. The large solenoid is used to move two phalanges simultaneously and the small solenoid is used to move a single phalange.

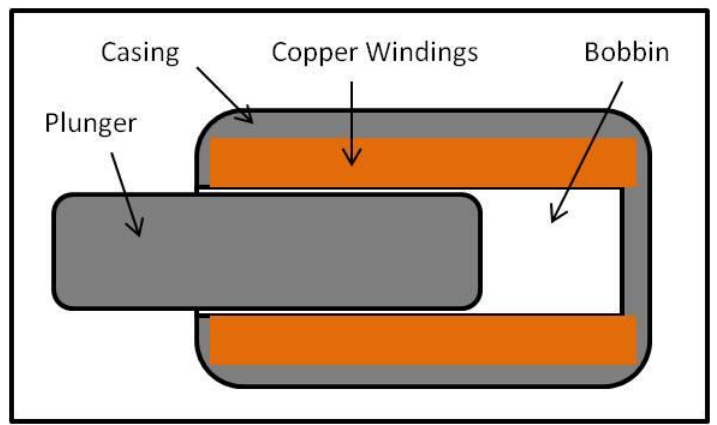

Figure 4. Basic solenoid actuator design

It is important to note that the double phalanges solenoid actuators are larger than the single phalange solenoid actuators because of two main reasons. The first reason is that the larger solenoid actuators have longer stroke length to move two phalanges simultaneously. Depending on certain mechanical factors, the stroke length requirement for moving two phalanges simultaneously is twice the amount of stroke length required for moving one phalange. The second reason is that the larger solenoid actuators can generate higher grasping strength. This statement can be easily understood in the next section which describes the basic motions of the finger.

\section{RESEARCH METHOD}

\subsection{Basic Finger Motions}

In order to generate motions for each finger (including the thumb) by using solenoid actuators, the proposed mechanism is based on a muscle-tendon-joints connection. The plunger, which is the moveable part of the solenoid actuator (muscle), will provide a pulling force on the high tension string (tendon) to bend the hinges (joints). The pulling force will then cause the hinges at the finger's joints to bend via the high tension string, which will subsequently move the finger into grasping position. The finger will return back to its original position once the plunger does not provide anymore pulling force on the high tension string because the bended hinge will return back to its resting position. Due to the solenoid actuator's limited stroke length, each finger requires two solenoid actuators to generate full motion (under-actuated) as shown in Figure 5. 


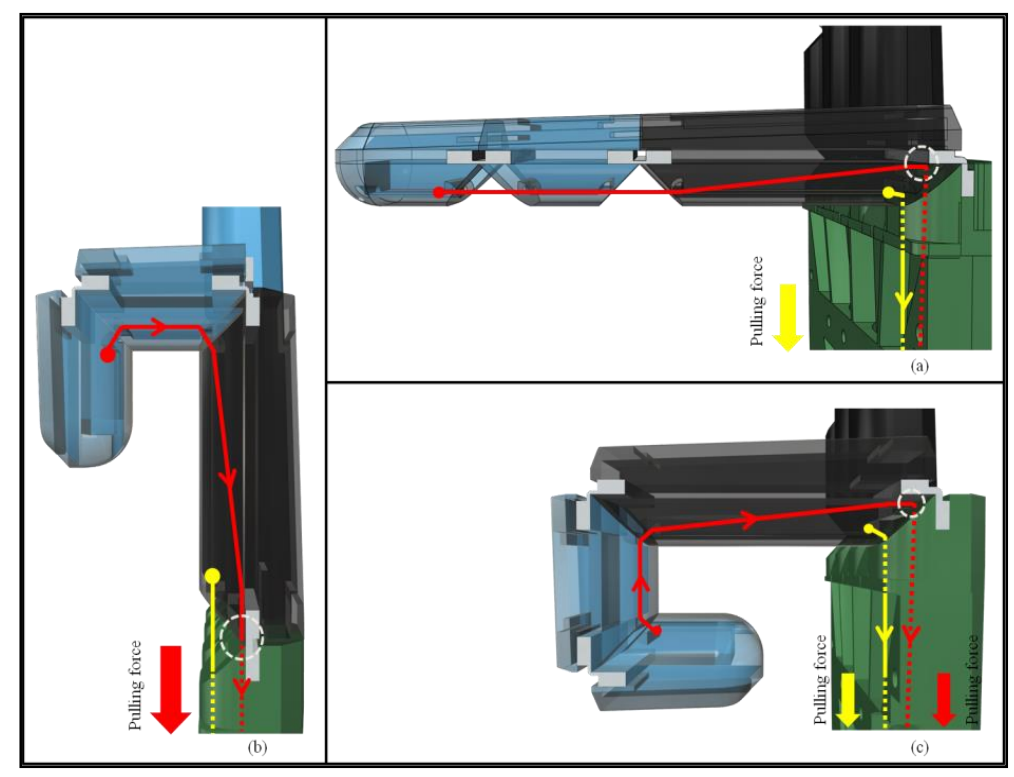

Figure 5. The finger's positions when the (a) single phalange solenoid actuator is activated,

(b) double phalanges solenoid actuator is activated and (c) both solenoid actuators are activated

As mentioned above, a single finger requires two solenoid actuators to create a complete motion because the total stroke length required to move three phalanges is $33.3 \mathrm{~mm}$ ( $11.1 \mathrm{~mm}$ for each phalange). Initially, the finger's position is shown in Figure 2(c). When the solenoid actuator for the single phalange is activated, it will pull the single phalange high tension string (yellow) and moves the bottom phalange. This phalange will move because the high tension string is tied to the single phalange string stopper as illustrated in Figure 5(a).

Take note of the dotted circles in the figure which emphasise the double phalanges high tension string's (red) placement that is very near to the hinge's pivot. This particular placement is important to ensure that the top and middle phalanges' movements are not critically affected when the bottom phalange moves. However, the double phalanges high tension string will be slightly loosen when the bottom phalange moves from finger's position in Figure 2(c) to finger's position in Figure 5(a) because the string's location is not exactly at the pivot.

Conversely, when the solenoid actuator for the double phalanges is activated, it will pull the double phalanges high tension string and moves the top and middle phalanges to create a hooking action. These phalanges will move because the high tension string is tied to the double phalange string stopper as illustrated in Figure 5(b).

The finger will create a complete motion when both solenoid actuators are activated to move the three phalanges as illustrated in Figure 5(c). Again, these phalanges will move because the high tension strings are tied to the string stoppers.

Based on the finger's motions, the grasping or holding strength is mostly depends on the net force of the double phalanges solenoid actuators. This is due to the fact that the hooking position is already sufficient to grab or hold an object. Therefore, the double phalanges solenoid actuators must have a larger net force as compared to the single phalange solenoid actuators. Nevertheless, the single phalange solenoid actuators are still important to assist in grasping objects that have various shapes and sizes.

\subsection{The Five-Fingered Robotic Hand Prototype}

The prototype, which is shown in Figure 6 without covers, was used to evaluate the solenoid actuators' capability in generating basic finger motions as described in Section 2.1.

This prototype's appearance is similar to the graphical design because the parts are 3D printed. Only the solenoid actuators, high tension strings and string stoppers were acquired in readymade forms. To be presice, the solenoid actuators are made by Guardian Electric and rated at $12 \mathrm{~V}$ but the single phalange solenoid actuators use the T6x12-I-12 model (top) that has a maximum stroke length of $12.7 \mathrm{~mm}$ whereas the double phalanges solenoid actuators use the bigger T8X16-I-12 model (bottom) that has a maximum stroke length of $25.4 \mathrm{~mm}$. Meanwhile, the high tension strings and string stoppers are made from braided nylon fishing line and plastic bead respectively. 


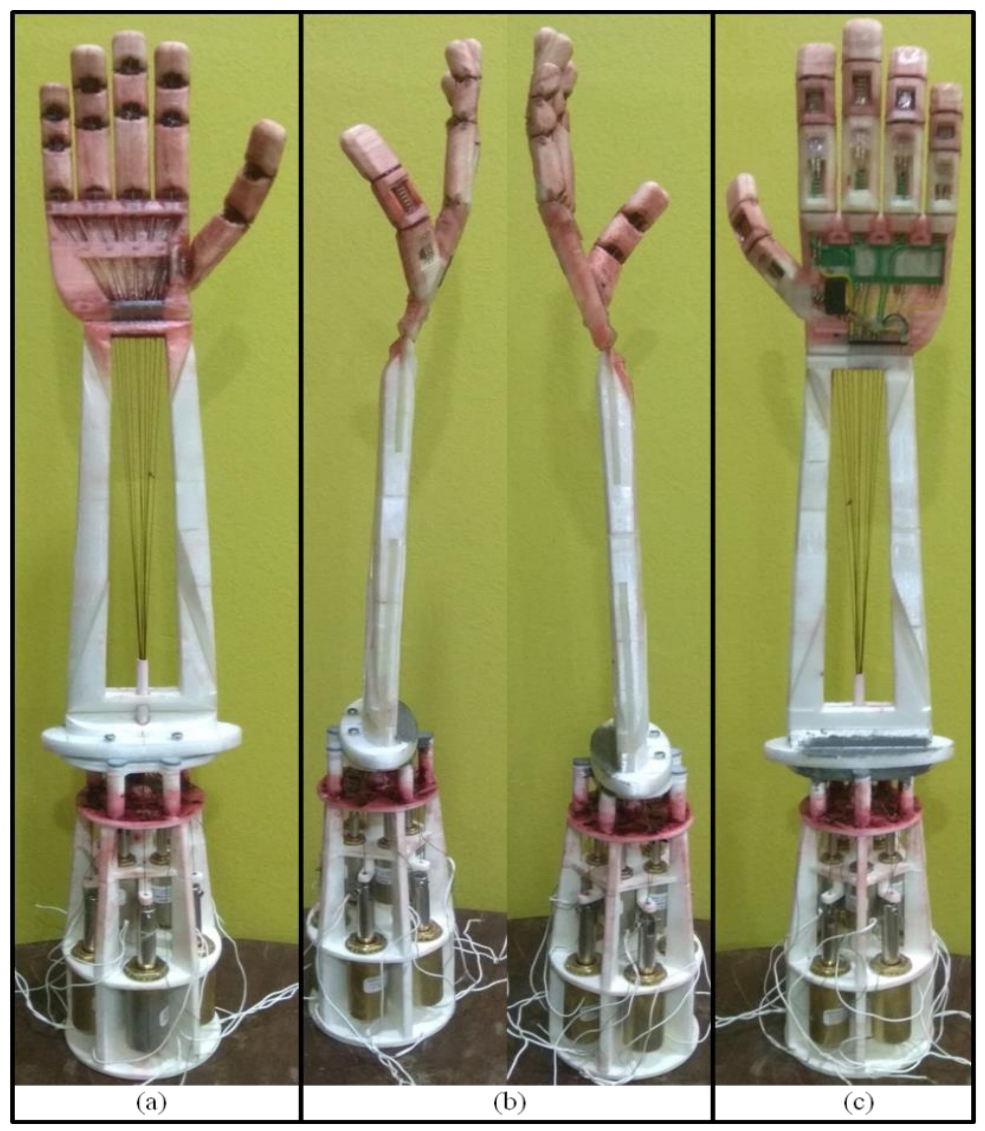

Figure 6. The five-fingered robotic hand prototype (a) palm, (b) sides and (c) backhand view

\section{RESULTS AND ANALYSIS}

The prototype's basic finger motions, which are based on the operational concept described in Section 2.1, are depicted in Figure 7.

It can be seen that the prototype's basic finger motions are similar to the basic finger motions of the design. Even though there is a slight angle variation due to some unexpected mechanical factors such as inconsistent hinge stiffness and string tension, the phalanges moved in accordance to the assumed or expected positions of the design. Therefore, these outcomes have proven that the solenoid actuators are capable of generating basic finger motions for a five-fingered robotic hand.

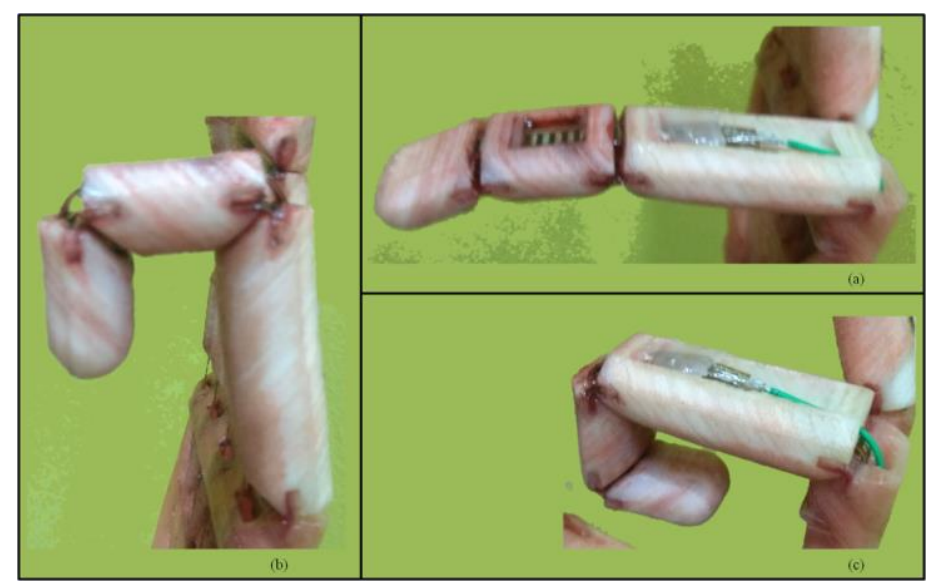

Figure 7. The prototype finger's positions when the (a) single phalange solenoid actuator is activated,

(b) double phalanges solenoid actuator is activated and (c) both solenoid actuators are activated 


\section{CONCLUSION}

Due to the similarity between the basic finger motions that were assumed in the design and also the prototype's perceptible outcomes, it can be concluded that the solenoid actuators have the capability to create basic fingers' motions. However, further research and developments are required to gauge the solenoid actuators' true potentials in the aspect of five-fingered robotic hand design such as grasping strength, reaction time and dexterity. This can be realised through in-depth calculations, simulations and further experimentations. Nevertheless, these findings can become a catalyst for future improvements.

\section{ACKNOWLEDGEMENTS}

The authors would like to thank the Malaysian government for funding this project in 2013 until 2016 under the Exploratory Research Grant Scheme (ERGS): 06012013ERGS.

\section{REFERENCES}

[1] "Robotics". Oxford Dictionaries. Retrieved 20 February 2013.

[2] V. Aryai, M. Kharazi and F. Ariai, "“Nearest Zero-Point" Algorithm for Cooperative Robotic Search Missions", International Journal of Robotics and Automation (IJRA), vol. 11, no. 4, pp. 25-38, 2017.

[3] U. Qidwai, M. Akbar, M. Maqbool and M. Jahanshahi, "Hierarchical Inspection System Using Visual and MFL Probe Robots", International Journal of Robotics and Automation (IJRA), vol. 7, no. 4, pp. 283-296, 2018.

[4] M. Mujiarto et al., "Colored Object Detection using 5 Dof Robot Arm based Adaptive Neuro-Fuzzy Method", Indonesian Journal of Electrical Engineering and Computer Science (IJEECS), vol. 13, no. 1, p. 293, 2019. Available: 10.11591/ijeecs.v13.i1.pp293-299.

[5] B. Suthar and N. Sindhu, "Design of Energy Efficient Four Finger Robotic Hand", International Journal of Robotics and Automation (IJRA), vol. 5, no. 1, pp. 1-5, 2016.

[6] W. Yan, H. Nie, J. Chen and D. Han, "Optimal Design and Grasp Ability Evaluation of Four-Finger Tendon-Driven Hand", International Journal of Advanced Robotic Systems, vol. 14, no. 6, p. 172988141774844, 2017. Available: $10.1177 / 1729881417748444$.

[7] G. Carbone, C. Rossi and S. Savino, "Performance Comparison Between FEDERICA Hand and LARM Hand", International Journal of Advanced Robotic Systems, vol. 12, no. 7, p. 90, 2015. Available: 10.5772/60523.

[8] Palli, G.; Scarcia, U.; Melchiorri, C.; Vassura, G.; , "Development of Robotic Hands: The UB hand evolution," Intelligent Robots and Systems (IROS), 2012 IEEE/RSJ International Conference on , vol., no., pp.5456-5457, 7-12 Oct. 2012.

[9] Shengqi Tan; Wenzeng Zhang; Qiang Chen; Dong Du; , "Design and Analysis of Underactuated Humanoid Robotic Hand based on Slip Block-Cam Mechanism," Robotics and Biomimetics (ROBIO), 2009 IEEE International Conference on , vol., no., pp.2356-2361, 19-23 Dec. 2009.

[10] Sonoda, T.; Godler, I.; , "Multi-Fingered Robotic Hand Employing Strings Transmission Named "Twist Drive" Video Contribution," Intelligent Robots and Systems (IROS), 2010 IEEE/RSJ International Conference on , vol., no., pp.2527-2528, 18-22 Oct. 2010.

[11] Ishii, C.; Nishitani, Y.; Hashimoto, H.; , "Robotic Hand with A New Bending Mechanism," Mechatronics and Automation, 2009. ICMA 2009. International Conference on , vol., no., pp.32-36, 9-12 Aug. 2009.

[12] Schmitz, A.; Pattacini, U.; Nori, F.; Natale, L.; Metta, G.; Sandini, G.; , "Design, Realization and Sensorization of The Dexterous iCub Hand," Humanoid Robots (Humanoids), 2010 10th IEEE-RAS International Conference on , vol., no., pp.186-191, 6-8 Dec. 2010.

[13] Deshpande, A.D.; Zhe Xu; Weghe, M.J.V.; Brown, B.H.; Ko, J.; Chang, L.Y.; Wilkinson, D.D.; Bidic, S.M.; Matsuoka, Y.; , "Mechanisms of the Anatomically Correct Testbed Hand," Mechatronics, IEEE/ASME Transactions on, vol.18, no.1, pp.238-250, Feb. 2013.

[14] Shadow Robot Company Ltd., "Shadow Dexterous Hand Technical Specification," London, 2013.

[15] Kyu-Jin Cho; Rosmarin, J.; Asada, H.; , "SBC Hand: A Lightweight Robotic Hand with an SMA Actuator Array implementing C-segmentation," Robotics and Automation, 2007 IEEE International Conference on , vol., no., pp.921-926, 10-14 April 2007.

[16] Kyu-Jin Cho; Rosemarin, J.; Asada, H.; , "Design of Vast DOF Artificial Muscle Actuators with A Cellular Array Structure and its Application to A Five-Fingered Robotic Hand," Robotics and Automation, 2006. ICRA 2006. Proceedings 2006 IEEE International Conference on , vol., no., pp.2214-2219, 15-19 May 2006.

[17] ]X. An, X. Liu and B. Sun, "Dynamic Characteristics of the Nozzle Opening Pressure based on the Fluid-Structure Interaction for A Double-Solenoid-Valve Fuel Injection System", Proceedings of the Institution of Mechanical Engineers, Part D: Journal of Automobile Engineering, vol. 231, no. 11, pp. 1589-1602, 2016. Available: 10.1177/0954407016680395.

[18] Figliolini, G.; Rea, P.; , "Ca.U.M.Ha. Robotic Hand (Cassino-Underactuated-Multifinger-Hand)," Advanced Intelligent Mechatronics, 2007 IEEE/ASME international conference on , vol., no., pp.1-6, 4-7 Sept. 2007.

[19] N. Hung and O. Lim, "A Simulation and Experimental Study on the Operating Characteristics of A Solenoid Gas Injector", Advances in Mechanical Engineering, vol. 11, no. 1, p. 168781401881742, 2019. Available: $10.1177 / 1687814018817421$. 
[20] Tan, L.Q.; Xie, S.Q.; Lin, I.C.; Lin, T.; , "Development of A Multifingered Robotic Hand," Information and Automation, 2009. ICIA '09. International Conference on , vol., no., pp.1541-1545, 22-24 June 2009.

[21] Gaiser, I.; Schulz, S.; Kargov, A.; Klosek, H.; Bierbaum, A.; Pylatiuk, C.; Oberle, R.; Werner, T.; Asfour, T.; Bretthauer, G.; Dillmann, R.; , "A New Anthropomorphic Robotic Hand," Humanoid Robots, 2008. 8th IEEE-RAS International Conference on, vol., no., pp.418-422, 1-3 Dec. 2008.

[22] Situm, Z.; Zilic, T.; Essert, M.; , "High Speed Solenoid Valves in Pneumatic Servo Applications," Control \& Automation, 2007. MED '07. Mediterranean Conference on , vol., no., pp.1-6, 27-29 June 2007.

[23] Qilei Wang, Fengyu Yang, Qian Yang, Junhui Chen, Hongyan Guan, "Experimental Analysis of New High-Speed Powerful Digital Solenoid Valves", Energy Conversion and Management, Volume 52, Issue 5, May 2011, Pages 2309-2313, ISSN 0196-8904, 10.1016/j.enconman.2010.12.032.

[24] So-Nam Yun; Hwang-Hoon Jeong; Soon-Chan Hwang; Hyo-Bong Kim; In-Seop Park; , "High Speed Solenoid Actuator for High-Voltage Circuit Breaker," Control, Automation and Systems (ICCAS), 2011 11th International Conference on, vol., no., pp.1006-1010, 26-29 Oct. 2011.

[25] J. Horner, "Electrical Controls," in Automotive electrical handbook. United States of America: HPBooks, 1987, ch. 5, pp. 33-34.

[26] S. Z. A. S. K. Bahrin, "Design and Development of a New Electromagnetic Prime Mover Using Solenoid Technology," Kajang, Selangor, 2011.

[27] K. Mitsui, R. Ozawa and T. Kou, "An Under-Actuated Robotic Hand for Multiple Grasps," 2013 IEEE/RSJ International Conference on Intelligent Robots and Systems, Tokyo, 2013, pp. 5475-5480.

[28] T. R. Hunt, C. J. Berthelette and M. B. Popovic, "Linear One-to-Many (OTM) system," Technologies for Practical Robot Applications (TePRA), 2013 IEEE International Conference on, Woburn, MA, 2013, pp. 1-6.

[29] B. Shields and M. Goldfarb, "Design and Energetic Characterization of a Solenoid Injected Liquid Monopropellant Powered Actuator for Self-Powered Robots," Proceedings of the 2005 IEEE International Conference on Robotics and Automation, 2005, pp. 241-246.

[30] S. Obata, T. Haneyoshi and Y. Saito, "New Linear Solenoid Actuator for Humanoid Robot," Mecatronics (MECATRONICS), 2014 10th France-Japan/ 8th Europe-Asia Congress on, Tokyo, 2014, pp. 367-370.

[31] A. Takai, N. Alanizi, K. Kiguchi and T. Nanayakkara, "Prototyping the Flexible Solenoid-Coil Artificial Muscle, for Exoskeletal Robots," Control, Automation and Systems (ICCAS), 2013 13th International Conference on, Gwangju, 2013, pp. 1046-1051.

[32] S. Z. A. S. K. Bahrin and K. S. M. Sahari, "Design and Development of A Five-Fingered Master-Slave Robotic Hand by using Solenoid and Pressure Sensors Comparator Technology Solenoid Actuation System," 2016 IEEE International Conference on Power and Energy (PECon), Melaka, 2016, pp. 495-499.

[33] "Human Engineering Design Data Digest". (2000). Washington, D.C.: The Group.

\section{BIOGRAPHIES OF AUTHORS}

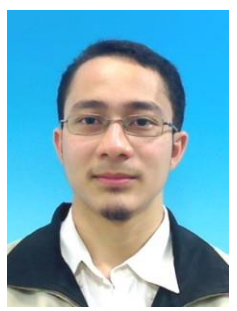

Syed Zainal Abidin Syed Kamarul Bahrin was born on January 22, 1984. He obtained his Bachelor of Electrical and Electronics Engineering (2006) and Master of Electrical Engineering (2012) at Universiti Tenaga Nasional, Malaysia. He is currently doing his $\mathrm{PhD}$ in Engineering that focuses on robotics at Universiti Tenaga Nasional, Malaysia. His topics of research include education, renewable energy, robotics, system automation, electrical machinery and automotive.

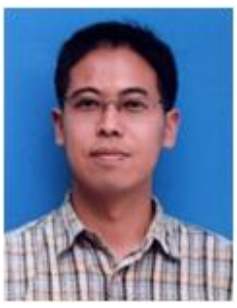

Khairul Salleh Mohamed Sahari: He is currently a professor at the Department of Mechanical Engineering, Universiti Tenaga Nasional. He received his PhD from Kanazawa University, Japan in 2006. His research interest includes robotics, mechatronics, Artificial Intelligence and Machine Learning. 